\title{
Reaction of HIV screening test for a renal cancer patient confirmed to be uninfected with HIV virus: a case report
}

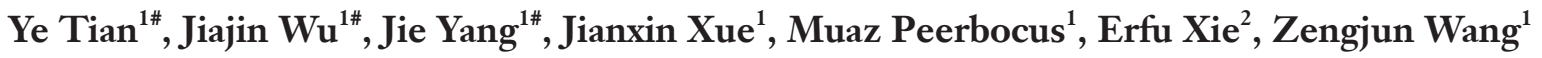 \\ ${ }^{1}$ Department of Urology, The First Affiliated Hospital of Nanjing Medical University, Nanjing, China; ${ }^{2}$ Department of Laboratory Medicine, The \\ First Affiliated Hospital of Nanjing Medical University, Nanjing, China \\ "These authors contributed equally to this work. \\ Correspondence to: Zengjun Wang. Department of Urology, the First Affiliated Hospital of Nanjing Medical University, No. 300 Guangzhou Road, \\ Nanjing, China. Email: zengjunwang@njmu.edu.cn; Erfu Xie. Department of Laboratory Medicine, the First Affiliated Hospital of Nanjing Medical \\ University, No. 300 Guangzhou Road, Nanjing, China. Email: xieerfu@njmu.edu.cn.
}

\begin{abstract}
Diagnosis of HIV infection and AIDS need to rely on HIV antibody or HIV antigen test internationally, and the test process is divided into preliminary screening test and confirmatory test. The HIV/AIDS screening laboratory of the First Affiliated Hospital of Nanjing Medical University has been using electrochemiluminescence (ECL) to screen the patients. A 50-year-old man with his enhanced computed tomography (CT) scan revealing two soft tissue density images were seen in the left kidney came to the hospital for medical treatment. The patient's postoperative pathological examination revealed (left kidney) renal clear cell carcinoma. The HIV screening tests of the patient several times in perioperative period showed reactivity, but the result of the confirmatory tests of Nanjing Municipal Center for Disease Control and Prevention were negative all the time. False positive HIV results are quite rare in the setting of renal clear cell carcinoma. There must be some substances that can react with the HIV Combi PT designed to detect anti-HIV antibodies of the IgG- and IgM-class as well as HIV p24 antigen in this patient. In conclusion, false positive results of HIV screening test may occur in serum of patients with renal cell carcinoma, and necessary confirmatory tests are needed. When clinicians encounter such problems, confirmatory tests should be conducted according to the guidelines to avoid misdiagnosis.
\end{abstract}

Keywords: AIDS; HIV; renal cell cancer; screening test; case report

Submitted Apr 30, 2020. Accepted for publication Sep 10, 2020.

doi: $10.21037 /$ tau-20-905

View this article at: http://dx.doi.org/10.21037/tau-20-905

\section{Introduction}

According to the third edition of National Guideline for Detection of HIV/AIDS (2015 edition) in China, diagnosis of HIV infection and AIDS need to rely on HIV antibody or HIV antigen test, and the test process is divided into preliminary screening test and confirmatory test. The preliminary screening test generally uses electrochemiluminescence (ECL), ELISA, immunofluorescence and immune colloidal gold technique, while the confirmatory test usually uses western blotting. The HIV/AIDS screening laboratory of the First Affiliated Hospital of Nanjing Medical University has been using
ECL to screen the patients. The HIV screening tests carried out by this patient several times in our hospital within 1 year showed reactivity, but the result of the confirmatory tests was negative all the time. This rare phenomenon in clinic is reported as follows.

We present the following article in accordance with the CARE reporting checklist (available at http://dx.doi. org/10.21037/tau-20-905).

\section{Case presentation}

A 50-year-old Asia man with his contrast-enhanced computed tomography (CT) scan revealing two soft tissue 


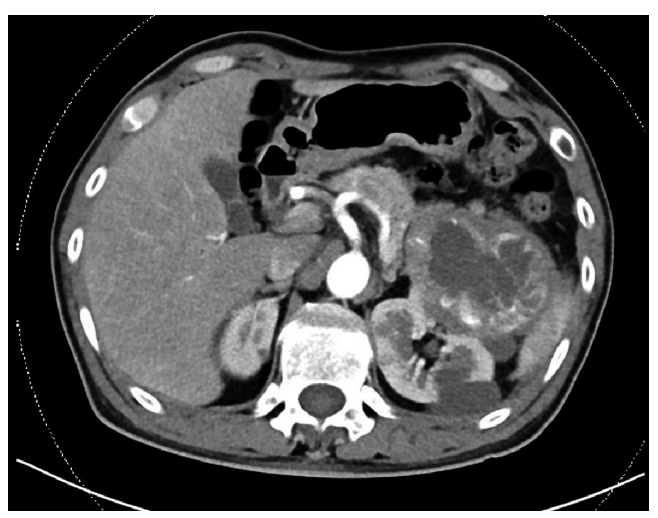

Figure 1 CT image revealing two soft tissue density images were seen in the left kidney. CT, computed tomography.

density images were seen in the left kidney came to our hospital for medical treatment, and the tissues' sizes were $7.8 \mathrm{~cm} \times 8.1 \mathrm{~cm} \times 7.0 \mathrm{~cm}$ and $1.5 \mathrm{~cm} \times 1.3 \mathrm{~cm}$, respectively since January 2018 (Figure 1). A large space-occupying lesion of the upper pole of the left kidney, possible renal carcinoma involving the tail of the pancreas, possibility of adjacent peritoneum metastasis. On admission, his physical examination and laboratory data were unremarkable. The patient reported an unremarkable medical, surgical, family history and no history of autoimmune diseases, blood diseases, infectious diseases or blood transfusion.

The preoperative examination showed that the patient's serum HIV COI value was 1.37 , and the result was reactive according to the instruction of the HIV Combi PT of Roche Diagnostics GmbH. On the basis of the third edition of National Guideline for Detection of HIV/AIDS, the HIV/AIDS screening laboratory of our hospital tested the sample again. And the HIV COI value was 1.33 , meaning it is reactive. Then the primary screening laboratory needs to use different methods of principle or reagents from different manufacturers for the third test. The third test using colloidal gold of Intec Products Incorporation showed nonreactive. The preliminary screening results meet the requirements of further confirmatory experiments. After confirmation of the patient's identity, another specimen was collected from the patient. Finally, we sent the two samples to Nanjing Municipal Center for Disease Control and Prevention (Nanjing CDC) for the west blotting. The MP Diagnostics (MPD) HIV BLOT 2.2 has been adopted by Nanjing CDC. While waiting for the result of the confirmatory test, the patient decided to leave hospital temporarily. At last the outcome from Nanjing CDC is negative (Table 1).

Therefore, we immediately informed the patient of the final result. The patient was admitted to hospital for surgical treatment. Surprisingly, according to regular procedure, the preoperative examination showed that the patient's third serum HIV COI value was 1.41. It meant that the patient still had the risk of contracting AIDS. Then the sample was tested successively by the HIV Combi PT of Roche Diagnostics GmbH and the colloidal gold of Intec Products Incorporation. The results were separately 1.36 and nonreactive. Subsequently, the fourth blood sample was collected from the patient the same day. Finally, the both blood samples were sent to Nanjing CDC again. This time we chose to take the risk of being infected to operate on this patient who may be infected with AIDS. The robot-assisted laparoscopic radical nephrectomy of left renal was carried out, and pathological examination revealed (left kidney) renal clear cell carcinoma, Grade II, T2bM0N0; Mass 4, size: $0.5-7.5 \mathrm{~cm} \times 7.5 \mathrm{~cm} \times 7 \mathrm{~cm}$, focal cystic changes in the tumor; no cancerous involvement in the renal pelvis. There are more than 10 simple renal cysts in the surrounding renal tissues, size: $0.2-0.7 \mathrm{~cm}$. The cancerous tissue did not break through the renal capsule (Figure 2). After several days, the outcome from Nanjing CDC was still negative. The patient tolerated the procedure well and there were no adverse or unanticipated events (Figure 3).

All procedures performed in studies involving human participants were in accordance with the ethical standards of the institutional and/or national research committee(s) and with the Helsinki Declaration (as revised in 2013). Written informed consent was obtained from the patient.

\section{Discussion}

HIV infection has evolved from a highly lethal disease to a chronic illness commonly managed in the primary care setting. False positive HIV results has been reported in a myriad of different settings-schistosomiasis, systemic lupus erythematosus, influenza vaccination. The patient proved HIV-negative twice by the confirmatory tests of Nanjing CDC. But all the results of the HIV Combi PT were reactive indeed. To our knowledge, there must be some substances that can react with the HIV Combi PT in this patient. The following are some possible causes of this phenomenon.

(I) The Elecsys ${ }^{\circledR}$ HIV Combi PT assay is designed to detect anti-HIV antibodies of the IgG- and IgMclass as well as HIV p24 antigen, which could 
Table 1 Preliminary screening and confirmed results of three samples of the patient

\begin{tabular}{|c|c|c|c|c|}
\hline Date of specimen collection & Test method & Test date & Result & Normal reference range \\
\hline \multirow{4}{*}{ 2018/1/31 } & Elecsys & $2018 / 1 / 31$ & 1.37 & $0-1$ \\
\hline & Elecsys & $2018 / 1 / 31$ & 1.33 & $0-1$ \\
\hline & Colloidal gold method & $2018 / 1 / 31$ & Negative & Negative \\
\hline & WB & $2018 / 2 / 8$ & Negative & Negative \\
\hline \multirow[t]{4}{*}{$2018 / 2 / 26$} & Screening test & & & \\
\hline & Elecsys & $2018 / 2 / 26$ & 1.41 & $0-1$ \\
\hline & Elecsys & $2018 / 2 / 26$ & 1.36 & $0-1$ \\
\hline & WB & $2018 / 3 / 1$ & Negative & Negative \\
\hline
\end{tabular}

WB, western blot.

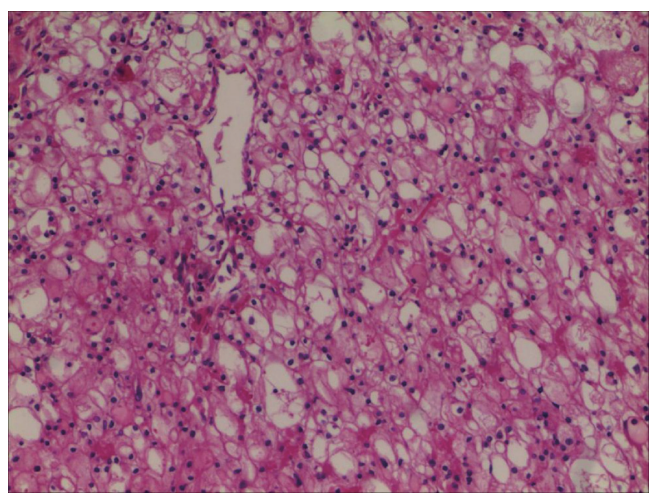

Figure 2 Postoperative pathological examination revealed (left kidney) renal clear cell carcinoma. Based on hematoxylin and eosin staining. Magnification: $\times 100$.

catch the HIV antigen and show reactive results in the early phase of HIV infection. The updated assay incorporates many improvements such as: pre-treatment step to increase specificity and sensitivity for detection of HIV-1 p24 antigen; a set of anti-p24 antibodies to provide high sensitivity for HIV-1 p24 antigen and allow detection of early infection, end-stage infection and p24 antigen derived from HIV-1 group O as well as HIV-2 (1). The specimen is incubated with a biotin
anti-P24/HIV recombinant antigen/HIV-specific polypeptide monoclonal antibody, and a ruthenium complex-labeled anti-P24/HIV recombinant antigen/HIV-specific polypeptide monoclonal antibody is added to the reaction. An antigenantibody complex is formed. Further, streptavidincoated microparticles are added, and the complex forms a solid phase under the interaction of streptavidin and biotin. The reaction solution is then sucked into the detection cell, and the particles in the detection cell are adsorbed on the surface of the electrode by electromagnetic action, and the unbound substance is removed by ProCell. A certain voltage is applied to the electrodes to cause the composite to chemiluminescence, and the intensity of the luminescence is detected by a photomultiplier. The intensity of the optical signal generated by the ECL process is linearly related to the concentration of divalent trichloro bipyridyl ruthenium $\left[\mathrm{Ru}(\mathrm{bpy})_{3}\right]^{2+}$, and the result of the increase in the intensity of the optical signal is reactive. Next, a few cases have been reported that patients treated with super-physiological dosage of biotin with subsequently abnormal hormone levels as a result of interference of excessive circulating biotin levels with the streptavidin-biotin-containing 

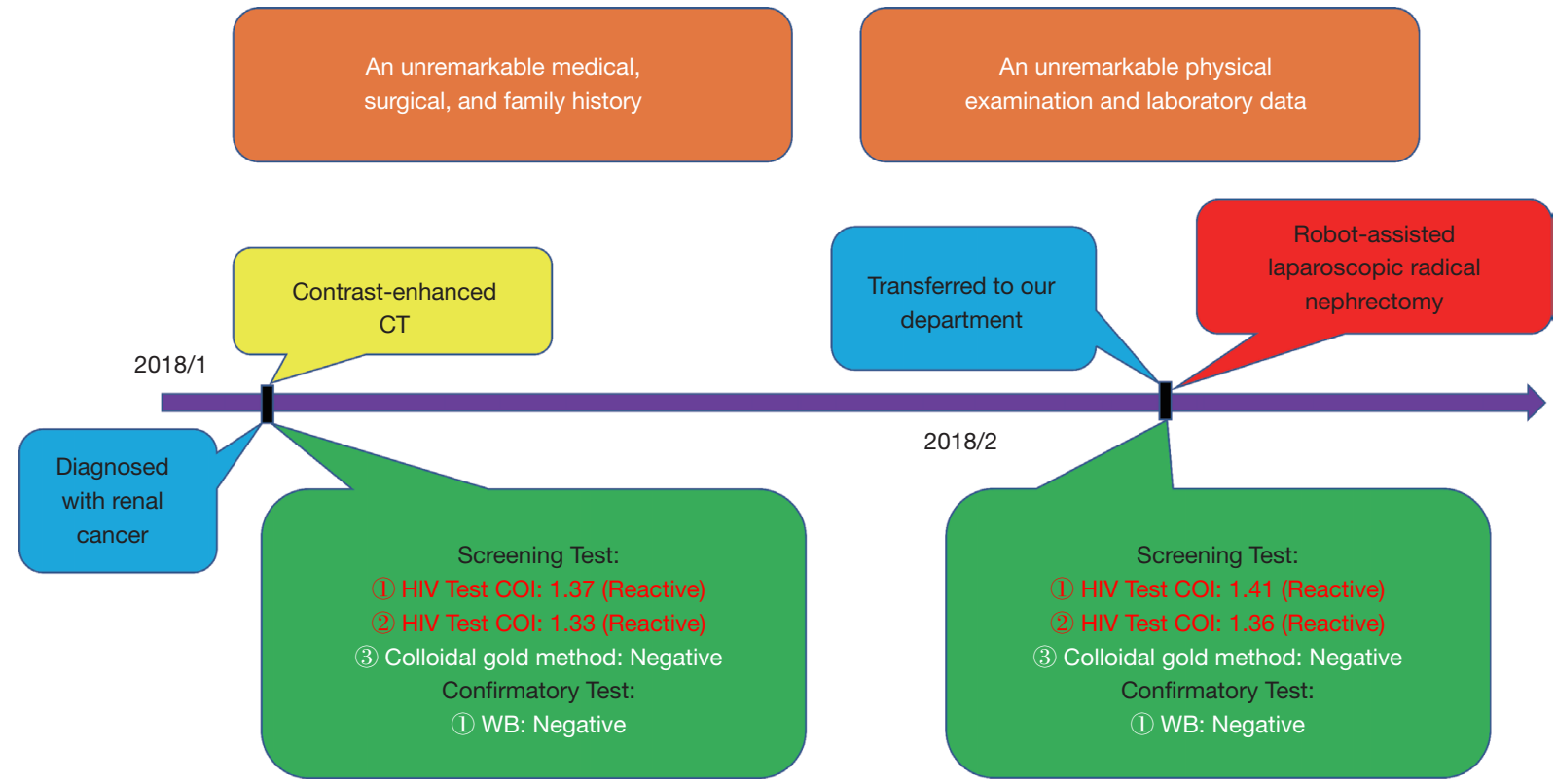

Figure 3 The timeline of the disease course. CT, computed tomography.

immunoassays (2-5). Therefore, treatment with ruthenium agent or over-dose biotin may result in a reactive result, but the patient has never before taken ruthenium agent or biotin.

(II) Some antigens in patients may contain common antigenic determinants of HIV antibodies, which results in cross reactions. Since the anti-HIV p24 antibody coated on the solid carrier is a murine antibody, some of the false reactions are due to the human anti-mouse antibody (HAMA) response.

(III) Some diseases can lead patients to contain some therapeutic antibodies, heterophil antibodies, rheumatoid factors, etc. These substances bound to the antigen components in the reagent nonspecific non-specifically may lead to false positives (6).

(IV) The patient maybe infected early with AIDS and has not yet produced antibodies. Because the ECL method of the preliminary screening test detects both HIV antigen and HIV antibody (1), while the immunoblot method of the confirmatory test only detects HIV antibody.

It has been reported that a female patient with severe malaria has a false positive HIV result, whose HIV screening test was positive and the confirmatory test were all negative. After medical treatment, her condition improved and the HIV screening tests reverted to negative (7). We planned to recommend the renal cancer patient to perform HIV screening tests and CT scans, however, we failed to follow up the patient regularly because of poor patient compliance. Hence, we did not know whether the results of postoperative examination reverted to negative. When clinicians encounter such problems, confirmatory tests should be conducted as soon as possible in strict accordance with the guidelines in order to avoid misdiagnosis.

\section{Acknowledgments}

Funding: The study was supported by the National Natural Science Foundation of China (Grants Nos. 81270685 and 81771640); Project of Nanjing Science and Technology Committee (201605001).

\section{Footnote}

Reporting Checklist: The authors have completed the CARE reporting checklist. Available at http://dx.doi.org/10.21037/ tau-20-905

Conflicts of Interest: All authors have completed the ICMJE uniform disclosure form (available at http://dx.doi. 
org/10.21037/tau-20-905). The authors have no conflicts of interest to declare.

Ethical Statement: The authors are accountable for all aspects of the work in ensuring that questions related to the accuracy or integrity of any part of the work are appropriately investigated and resolved. All procedures performed in studies involving human participants were in accordance with the ethical standards of the institutional and/or national research committee(s) and with the Helsinki Declaration (as revised in 2013). Written informed consent was obtained from the patient.

Open Access Statement: This is an Open Access article distributed in accordance with the Creative Commons Attribution-NonCommercial-NoDerivs 4.0 International License (CC BY-NC-ND 4.0), which permits the noncommercial replication and distribution of the article with the strict proviso that no changes or edits are made and the original work is properly cited (including links to both the formal publication through the relevant DOI and the license). See: https://creativecommons.org/licenses/by-nc-nd/4.0/.

Cite this article as: Tian $\mathrm{Y}, \mathrm{Wu} \mathrm{J}$, Yang J, Xue J, Peerbocus M, Xie E, Wang Z. Reaction of HIV screening test for a renal cancer patient confirmed to be uninfected with HIV virus: a case report. Transl Androl Urol 2020;9(5):2281-2285. doi:10.21037/ tau-20-905

\section{References}

1. Wang T, Li D, Yan K, et al. Performance evaluation of a new fourth-generation $\mathrm{HIV} \mathrm{Ag/Ab} \mathrm{combination}$ electrochemiluminescence immunoassay - evaluation of a new HIV assay. Int J STD AIDS 2014;25:267-72.

2. Barbesino G. Misdiagnosis of graves' disease with apparent severe hyperthyroidism in a patient taking biotin megadoses. Thyroid 2016;26:860-3.

3. Meany DL, Jan de Beur SM, Bill MJ, et al. A case of renal osteodystrophy with unexpected serum intact parathyroid hormone concentrations. Clin Chem 2009;55:1737-9.

4. Kummer S, Hermsen D, Distelmaier F. Biotin treatment mimicking graves' disease. N Engl J Med 2016;375:704-6.

5. Wijeratne NG, Doery JC, Lu ZX. Positive and negative interference in immunoassays following biotin ingestion: a pharmacokinetic study. Pathology 2012;44:674-5.

6. Patel KK, Gronowski AM. Heterophile antibody interference in qualitative urine/serum hCG devices: case report. Clin Biochem 2016;49:729-31.

7. Stempel JM, Mora Carpio AL, Puga D, et al. False positive fourth generation HIV test in a patient with severe malaria. Int J Infect Dis 2019;83:86-7. 